\title{
Muonium in Superfluid Helium
}

\author{
E. Krasnoperov and E. Meilikhov \\ Kurchatov Institute for Atomic Energy, Moscow, 123182, Russia \\ R. Abela, D. Herlach, and E. Morenzoni \\ Paul Scherrer Institute, CH-5232 Villigen PSI, Switzerland \\ F. N. Gygax and A. Schenck \\ Institute for Intermediate Energy Physics, Eidgenössische Technische Hochschule Zurich, CH-5232 Villigen PSI, Switzerland \\ D. Eschenko \\ Institute for Nuclear Research, Moscow 117312, Russia
}

(Received 9 July 1992)

\begin{abstract}
We present the first muon-spin-relaxation studies in superfluid ${ }^{4} \mathrm{He}$ in the temperature range $0.5-1.5$ $\mathrm{K}$. At temperatures below $1 \mathrm{~K}$ we observed muonium spin precession. From the magnetic-field dependence of the amplitude of the muonium component we estimate a muonium formation rate of about $5 \times 10^{7} \mathrm{~s}^{-1}$ at $0.5 \mathrm{~K}$. We find that external electric fields, preventing the convergence of muon and electron, reduce the amplitude of the muonium precession signal.

PACS numbers: $67.40 . Y v, 66.10 .-x, 76.75 .+\mathrm{i}$
\end{abstract}

The previous unsuccessful attempts to detect muonium atoms $\left(\mu^{+} e^{-}\right)$in gaseous [1] and liquid [2] helium at 4.2 and $2 \mathrm{~K}$ were ascribed to the high ionization potential of the He atom $\left(E_{i}=25 \mathrm{eV}\right)$ compared with the binding energy of muonium $\left(E_{b}=13.5 \mathrm{eV}\right)$. In a magnetic field applied transversely to the initial $\mu^{+}$polarization the muon was found to precess with the Larmor frequency of a free muon and no sizable depolarization was observed [2]. In more recent studies a low and essentially temperature-independent depolarization rate for $\mu^{+}$in ${ }^{4} \mathrm{He}$ in the normal state was observed, whereas in the superfluid state the depolarization was found to increase rapidly with decreasing temperature below the $\lambda$ point [3]. Additionally, below the $\lambda$ point, a fast-relaxing component of the muon spin polarization was observed and it was suggested that the muon depolarization was caused by the interaction of the muon with an electron at a distance below $10^{-4}-10^{-5} \mathrm{~cm}$, produced during the muon thermalization, but no muonium signal was detected [4]. In this paper we report on the first direct muonium observation in superfluid helium. The amplitude of the muonium spin precession is found to be a strong function of applied electric and magnetic fields. The results can be understood in terms of delayed muonium formation arising from the combination of the $\mu^{+}$and an electron near the end of the slowing-down track.

Positive and negative ions have been widely used to probe the properties of superfluid helium and several investigations have been performed to study the state of these ions [5]. It is known that heavy positive ions in liquid helium attract the neighboring neutral atoms sufficiently to generate a high-density permanent cluster of $\mathrm{He}$ atoms around the ion (snowball) [6]. On the other hand, an electron in the liquid will become localized within a bubble [7]. Because of the intermediate mass of the $\mu^{+}$(small in comparison to heavy ions, large in comparison to the electron), the state of the $\mu^{+}$-He complex in liquid $\mathrm{He}$ is a priori not well defined, but from a hydrodynamic point of view all charged-particle complexes are expected to have effective masses of several tens of He atoms and move according to the equations of viscous motion [6].

The mobility of charges in superfluid helium has been investigated extensively. Below the $\lambda$ point it rises exponentially with decreasing temperature and at $0.6 \mathrm{~K}$ $b_{+}=1700 \mathrm{~cm}^{2} \mathrm{~V}^{-1} \mathrm{~s}^{-1}$ and $b_{-}=155 \mathrm{~cm}^{2} \mathrm{~V}^{-1} \mathrm{~s}^{-1}$ for positive and negative charges, respectively $[8,9]$. The observation of long-lived muonium in superfluid $\mathrm{He}$ may therefore be ascribed to the huge mobilities in the superfluid state which allow the thermalized $\mu^{+}$and free electrons near the end of the $\mu^{+}$track to combine sufficiently fast to allow the evolution of phase-coherent muonium precession.

The experiments were performed at the surface muon beam channel $\pi \mathrm{M} 3$ of PSI utilizing the PSI $\mu$ SR-facility spectrometer [10] in conjunction with a continuous-flow ${ }^{3} \mathrm{He}$ cryostat, designed and built at the Kurchatov Institute for Atomic Energy, which allowed us to use temperatures down to $0.5 \mathrm{~K}$. The liquid ${ }^{4} \mathrm{He}$, in which the $\mu^{+}$ were stopped, was held in a 20-mm-diam, 9-mm-long copper cell, with an entrance window on one side made of 0.1 -mm-thick Kapton foil. A 5-8- $\mu$ m-thick copper foil in front of this window served as an electrode for the generation of an electric field in parallel to the $\mu^{+}$beam in the sample region. Transverse field muon-spin-relaxation $(\mu \mathrm{SR})$ spectra were recorded using conventional time differential techniques. The muons were stopped quantitatively in the liquid $\mathrm{He}$ and no signal from $\mu^{+}$stopped 
in the $\mathrm{Cu}$ cell could be seen.

Measurements in a field of $300 \mathrm{G}$ and above $1 \mathrm{~K}$ reproduced previous results [3]; a two-component signal is observed which is well described by the following expression for the time evolution of the muon's polarization:

$P(t) \propto\left[A_{f} \exp \left(-\lambda_{f} t\right)+A_{s} \exp \left(-\lambda_{s} t\right)\right] \cos \left(\omega_{\mu} t+\phi\right)$.

This expression consists of a fast-relaxing component with amplitude (equal to decay asymmetry) $A_{f}$ and relaxation rate $\lambda_{f}$ and a slowly relaxing component with $A_{s}$ and $\lambda_{s} . \omega_{\mu}=\gamma_{\mu} H_{\text {ext }}$ is the $\mu^{+}$Larmor frequency in the external field $H_{\text {ext. }} . \lambda_{f}$ is about 2 to 3 orders of magnitude larger than $\lambda_{s}\left(\lambda_{s}<0.3 \mu \mathrm{s}^{-1}\right)$. A significant fraction of the initial muon polarization is missing, as already observed in Ref. [4], which is strongly suggestive of slow muonium formation.

Indeed, by lowering the temperature below $1 \mathrm{~K}$ and using magnetic fields of less than $20 \mathrm{G}$, a long-lived muonium precession signal in the triplet spin state could be directly observed, whose gyromagnetic ratio is approximately 100 times that of a free muon and which approximately accounts for the missing polarization above $1 \mathrm{~K}$. In order to describe the data Eq. (1) has to be complemented as follows:

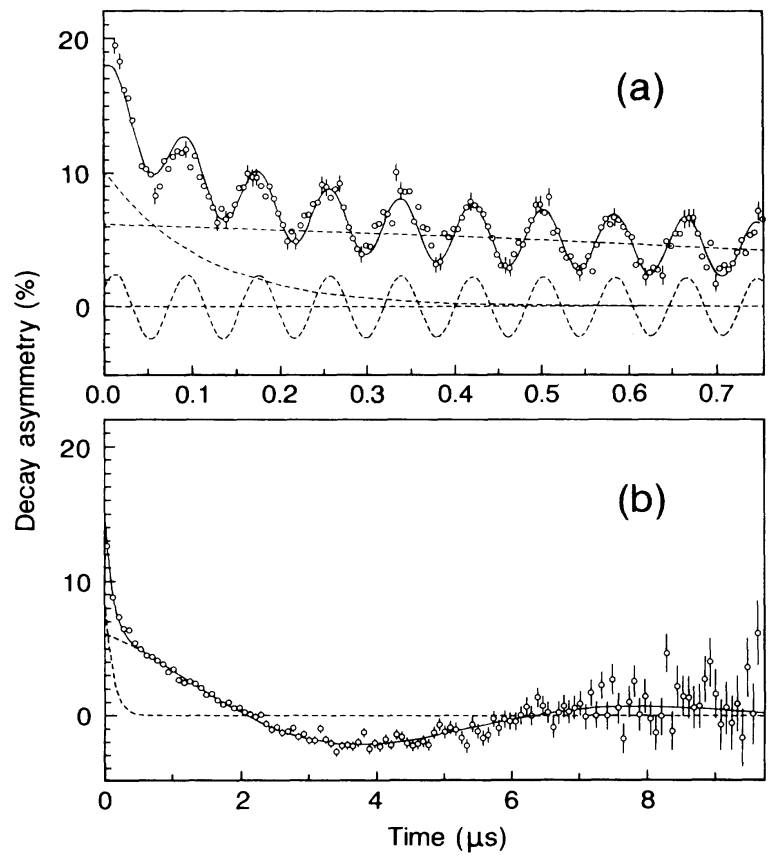

FIG. 1. Time evolution of $\mu^{+}$polarization in superfluid $\mathrm{He}$ at $0.52 \mathrm{~K}$ in an external field of $8.8 \mathrm{G}$. The solid line corresponds to a three-component fit [Eq. (2)] to the data, the dotted lines showing the individual components. (a) Early part of the spectrum, which shows clearly the muonium precession signal and the fast relaxing "free" $\mu^{+}$signal. (b) Full spectrum which shows the fast and slowly relaxing "free" $\mu^{+}$signals. The signal in (a) close to time zero indicates the presence of some additional very fast-relaxing component out of which the muonium signal has probably evolved.

$$
\begin{aligned}
P(t) \propto & {\left[A_{f} \exp \left(-\lambda_{f} t\right)+A_{s} \exp \left(-\lambda_{s} t\right)\right] } \\
& \times \cos \left(\omega_{\mu} t+\phi\right)+A_{\mathrm{Mu}} \exp \left(-\lambda_{\mathrm{Mu}} t\right) \cos \left(\omega_{\mathrm{Mu}} t+\phi^{\prime}\right) .
\end{aligned}
$$

$\omega_{\mathrm{Mu}} \simeq \frac{1}{2} m_{\mu} / m_{e} \gamma_{\mu} H_{\mathrm{ext}}$ is the muonium precession frequency in the triplet state in the linear Zeeman regime, i.e., $H_{\text {ext }} \ll 1585 \mathrm{G}$.

As an example Fig. 1 shows the $\mu \mathrm{SR}$ signal obtained at $0.52 \mathrm{~K}$ in a transverse field of $\sim 0.8 \mathrm{G}$. One notices the precession pattern of muonium in the triplet state [Fig. $1(\mathrm{a})$ ] and the fast and the slow polarization decay patterns of the diamagnetic (free) muon signal [Fig. 1(b)]. Not visible is the $50 \%$ fraction of muonium atoms in the singlet state which are associated with a frequency in the $\mathrm{GHz}$ range and which is not resolved by the spectrometer. $\lambda_{\mathrm{Mu}}$ is typically of the order of $0.1 \mu \mathrm{s}^{-1}$ at $0.52 \mathrm{~K}$.

The amplitude $A_{\mathrm{Mu}}$ of the muonium signal depends strongly on temperature, applied magnetic field, and also electrical field. $A_{\mathrm{Mu}}$ decreases with increasing temperature and is practically zero at $1.55 \mathrm{~K}$. Figure 2 shows the field dependence of $A_{\mathrm{Mu}}$ at $0.52 \mathrm{~K}$. It declines quickly with rising field and the muonium signal has disappeared for external fields $H_{\text {ext }} \gtrsim 20 \mathrm{G}$. Application of an electrical field parallel to the $\mu^{+}$beam likewise suppresses $A_{\mathrm{Mu}}$ as can be seen from Fig. 3. All three observations together suggest that one observes slow muonium formation by combination of initially free thermalized $\mu^{+}$and electrons created during the slowing down of the $\mu^{+}$close to the end of the $\mu^{+}$track.

Assuming for simplicity that the combination can be

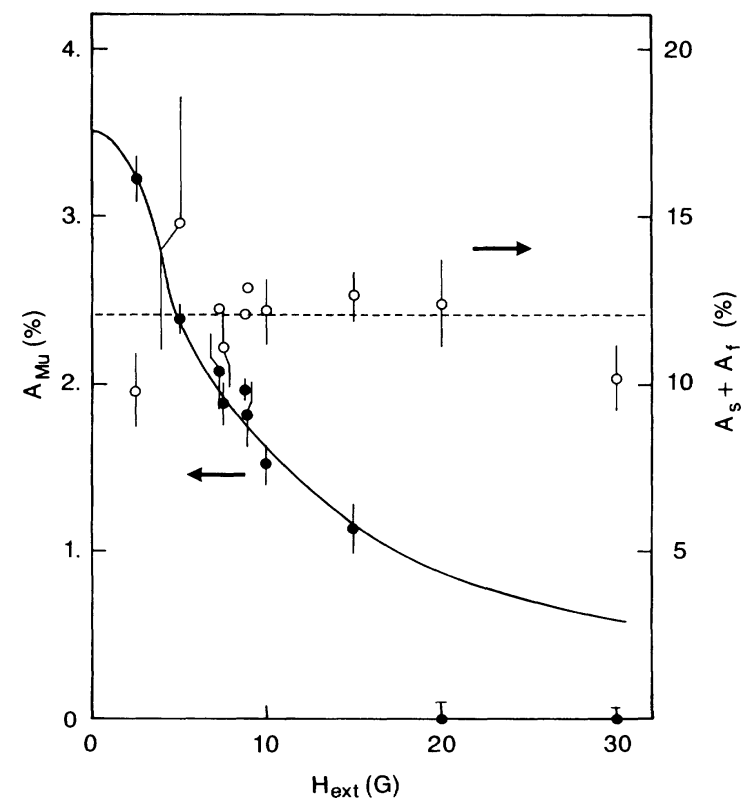

FIG. 2. Field dependence of the muonium amplitude $A_{\mathrm{Mu}}$ and the sum of the "free" $\mu^{+}$amplitudes $A_{s}$ and $A_{f}$ at $0.52 \mathrm{~K}$. The solid line is a fit of $A_{\mathrm{Mu}}$, defined in Eq. (4), to the data below $20 \mathrm{G}$. Note that $A_{s}+A_{f}$ appears to be field independent. 


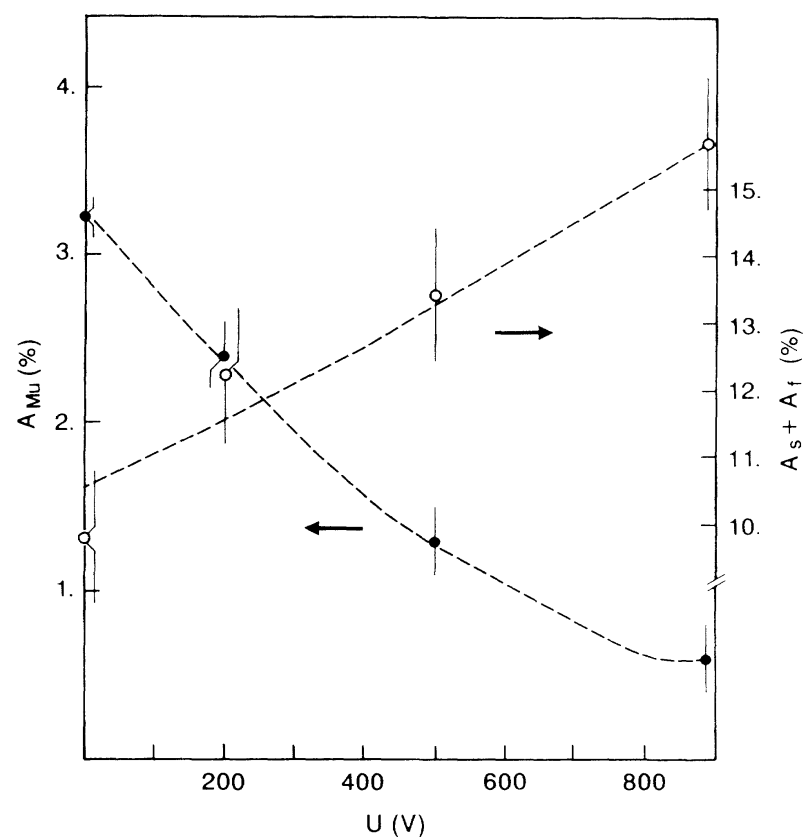

FIG. 3. Dependence of $A_{\mathrm{Mu}}$ and $A_{s}+A_{f}$ on an external electrical field at $0.52 \mathrm{~K}\left(H_{\mathrm{ext}}=2.5 \mathrm{G}\right)$.

described by a first-order kinetic equation (this approximation implies a time and space constant electron density which is certainly not realized in practice), the rate of muonium formation can be expressed as

$$
d n_{\mathrm{Mu}}(t)=-d n_{\mu}(t)=\lambda n_{\mu}(t) d t,
$$

i.e., $n_{\mu}(t)=n_{0} e^{-\lambda t}$, where $\lambda$ is the formation rate constant. The evolution of the $\mu^{+}$polarization, e.g., along the initial polarization, is then given by [11]

$$
\begin{aligned}
P(t)= & \frac{1}{2} P_{0} \frac{\lambda}{\left(\lambda^{2}+\omega_{\mathrm{Mu}}^{2}\right)^{1 / 2}}\left\{-e^{-\lambda t} \cos \varphi+\cos \left(\omega_{\mathrm{Mu}} t-\varphi\right)\right\} \\
& +P_{0} e^{-\lambda t} \cos \omega_{\mu} t
\end{aligned}
$$

where $\operatorname{tg} \varphi=\omega_{\mathrm{Mu}} / \lambda$ and $P_{0}$ denotes initial polarization. The first term refers to the muonium and the second term to the free muon signal. The factor $\frac{1}{2}$ accounts for the invisible spin-singlet muonium state. (Note that the polarized $\mu^{+}$combine with unpolarized electrons leading to an equal population of spin parallel and spin antiparallel configurations. Only the former one is an eigenstate of the hyperfine coupling Hamiltonian.) The field-dependent, generally reduced, amplitude $A_{\mathrm{Mu}}$ of the muonium signal is a result of dephasing as the muonium state is formed at different times and the phase coherence among the precessing muonium atoms is lost. The faster the muonium atoms precess, i.e., the larger $H_{\text {ext }}$, the faster the effect of dephasing. Note that $\omega_{\mathrm{Mu}} \gg \omega_{\mu}$ and that therefore the free muon precession has no effect on the dephasing. Equation (4) has almost the same form used to describe the data in Fig. 1. What is missing is the weakly damped free muon precession signal appearing in
Eqs. (1) and (2). The presence of this component implies that a certain fraction of $\mu^{+}$are not subject to muonium formation at least not within the time window accessible to $\mu \mathrm{SR}$.

Fitting the data in Fig. 2 by the formula for the amplitude of the muon signal in Eq. (4) one finds $\lambda=4.5 \times 10^{7}$ $\mathrm{s}^{-1}$ at $0.52 \mathrm{~K}$. The same value within $\pm 10 \%$ is found from an analysis of the relative phase shift of the muonium signal with respect to the free $\mu^{+}$signal $[\varphi$ in Eq. (4)]. The fit misses the faster drop of $A_{\mathrm{Mu}}$ above $15 \mathrm{G}$, indicating that Eq. (3) is perhaps an oversimplification. Nevertheless, $\lambda$ will be a good measure of the formation rate. The formation rate should also be deducible from the disappearance of the free muon signal, i.e., from the second component in Eq. (4) or the fast decay signal in the data. Direct fits to the data at $0.52 \mathrm{~K}$ yield a fieldindependent average value of $\lambda_{f} \simeq 1 \times 10^{7} \mathrm{~s}^{-1}$ which is significantly smaller than the value found from the field dependence of $A_{\mathrm{Mu}}$. In fact, if the effective $\lambda$ is as small as $10^{7} \mathrm{~s}^{-1}$ the muonium signal should already be reduced by more than $75 \%$ at $5 \mathrm{G}$, in contrast to what is observed. On the other hand, the disappearance of the diamagnetic $\mu^{+}$signal with a rate of $4.5 \times 10^{7} \mathrm{~s}^{-1}$ or decay time of 22 ns may have been masked by the initial dead time of the spectrometer. A hint for it may be seen in Fig. 1 (see caption). These considerations suggest that there are in fact two channels of muonium formation with significantly different rates. Altogether then, there would be at least three different channels of $\mu^{+}$behavior in superfluid He. It may be speculated that the different channels reflect different molecular ionic states of $\mu^{+}$(e.g., $\mu^{+} \mathrm{He}_{n}$ with different $n$ ) in liquid $\mathrm{He}$. Alternatively, the different channels may be explained in terms of the behavior of the track electrons in time and space. For example, if the electron density would drop to zero at a certain time after formation, the surviving free $\mu^{+}$would remain free and could account for the weakly damped diamagnetic $\mu^{+}$signal. In this respect it is interesting to note that $A_{s}+A_{f}$ in Fig. 3 increases almost by the same proportion as $2 A_{\mathrm{Mu}}$ (triplet + singlet $\mu^{+} e^{-}$) decreases with increasing voltage, quite in contrast to what is seen in Fig. 2. The decrease of $A_{\mathrm{Mu}}$ with voltage is therefore certainly not the result of a decrease of the formation rate $\lambda$ alone [see Eq. (4)] but at least in part of a genuine suppression of muonium formation and a consequently increased diamagnetic signal.

The effect of an external electrical field on the muonium formation process must be sought for in the competition of the Coulomb attraction between $\mu^{+}$and $e^{-}$and their interaction with the external field. However, a detailed understanding will rest on a better knowledge of the state(s) of the $\mu^{+}$molecular ionic complex, of the state(s) of the electrons created in the track, and of the involved kinetic behavior in superfluid He. The present results suggest that a study of the formation and motion of molecular ions in superfluid He by $\mu \mathrm{SR}$ spectroscopy is feasible and will constitute a challenge for future work. 
The authors are grateful to A. V. Driagin, S. T. Boldarev, and V. S. Mishachev for technical support.

[1] R. D. Stambaugh, D. E. Casperson, T. W. Crane, V. W. Hughes, H. F. Kaspar, P. Souder, P. A. Thompson, H. Orth, G. zu Putlitz, and A. B. Denison, Phys. Rev. Lett. 33, 568 (1974).

[2] T. W. Crane, D. E. Casperson, H. Chang, V. W. Hughes, H. F. Kaspar, B. Lovett, A. Schiz, P. Souder, R. D. Stambaugh, and G. zu Putlitz, Phys. Rev. Lett. 33, 572 (1974).

[3] E. P. Krasnoperov, V. G. Storchak, S. G. Barsov, A. L. Getalov, S. P. Kruglov, L. A. Kuz'min, S. M. Mikirtych'yants, and G. V. Shcherbakov, Pis'ma Zh. Eksp. Teor. Fiz. 42, 263 (1985) [JETP Lett. 42, 324 (1985)].

[4] D. G. Eschenko, E. P. Krasnoperov, S. G. Barsov, A. L. Getalov, L. A. Kuz'min, V. P. Koptev, S. M.
Mikirtych'yants, and G. V. Shcherbakov, Pis'ma Zh. Eksp. Teor. Fiz. 48, 568 (1988) [JETP Lett. 48, 616 (1988)].

[5] For a recent review see D. R. Tilley and J. Tilley, Superfluidity and Superconductivity (Adam Hilger, Bristol, 1990), and references therein.

[6] K. R. Atkins, Phys. Rev. 116, 1339 (1959).

[7] R. A. Ferrel, Phys. Rev. 108, 167 (1957).

[8] B. N. Eselson, Yu. Z. Kovdrja, and V. B. Shikin, Zh. Eksp. Teor. Fiz. 59, 64 (1970) [Sov. Phys. JETP 32, 37 (1970)]; D. M. Strayer, R. J. Donnelly, and P. H. Roberts, Phys. Rev. Lett. 26, 165 (1971).

[9] G. W. Rayfield, Phys. Rev. 168, 222 (1968).

[10] D. Herlach and I. D. Reid, in "Muon Spin Rotation Spectroscopy at the Swiss Institute for Nuclear Research (SIN), now Paul Scherrer Institute (PSI), Villigen, Switzerland," edited by A. Schenck, Paul Scherrer Institute Report No. PSI-TECDOC-1, 1990 (to be published), p. 306.

[11] P. W. Percival, E. Roduner, and H. Fischer, Chem. Phys. 32, 353 (1978). 